OPEN ACCESS

Edited by: Jian-Bing Fan,

Illumina, United States

Reviewed by: Lucia Tata-Chayeb, National Institute of Cancerology (INCAN), Mexico

Shanqiang Qu,

Southern Medical University, China

*Correspondence: Wei Zhang zn002646@whu.edu.cn

Specialty section: This article was submitted to Human and Medical Genomics,

a section of the journal

Frontiers in Genetics

Received: 29 June 2021 Accepted: 03 December 2021 Published: 04 January 2022

Citation:

Zhao K, Ma Z and Zhang W (2022) Comprehensive Analysis to Identify SPP1 as a Prognostic Biomarker in Cervical Cancer.

Front. Genet. 12:732822 doi: $10.3389 /$ fgene.2021.732822

\section{Comprehensive Analysis to Identify SPP1 as a Prognostic Biomarker in Cervical Cancer}

\author{
Kaidi Zhao, Zhou Ma and Wei Zhang* \\ Department of Obstetrics and Gynecology, Zhongnan Hospital of Wuhan University, Wuhan, China
}

Background: $S P P 1$, secreted phosphoprotein 1, is a member of the small integrinbinding ligand $\mathrm{N}$-linked glycoprotein (SIBLING) family. Previous studies have proven SPP1 overexpressed in a variety of cancers and can be identified as a prognostic factor, while no study has explored the function and carcinogenic mechanism of SPP1 in cervical cancer.

Methods: We aimed to demonstrate the relationship between SPP1 expression and pancancer using The Cancer Genome Atlas (TCGA) database. Next, we validated SPP1 expression of cervical cancer in the Gene Expression Omnibus (GEO) database, including GSE7803, GSE63514, and GSE9750. The receiver operating characteristic (ROC) curve was used to evaluate the feasibility of SPP1 as a differentiating factor by the area under curve (AUC) score. Cox regression and logistic regression were performed to evaluate factors associated with prognosis. The SPP1-binding protein network was built by the STRING tool. Enrichment analysis by the R package clusterProfiler was used to explore potential function of SPP1. The single-sample GSEA (sSGSEA) method from the $R$ package GSVA and TIMER database were used to investigate the association between the immune infiltration level and SPP1 expression in cervical cancer.

Results: Pan-cancer data analysis showed that SPP1 expression was higher in most cancer types, including cervical cancer, and we got the same result in the GEO database. The ROC curve suggested that SPP1 could be a potential diagnostic biomarker (AUC = 0.877). High SPP1 expression was associated with poorer overall survival (OS) $(P=0.032)$. Further enrichment and immune infiltration analysis revealed that high SPP1 expression was correlated with regulating the infiltration level of neutrophil cells and some immune cell types, including macrophage and DC.

Conclusion: SPP1 expression was higher in cervical cancer tissues than in normal cervical epithelial tissues. It was significantly associated with poor prognosis and immune cell infiltration. Thus, SPP1 may become a promising prognostic biomarker for cervical cancer patients.

Keywords: SPP1, biomarker, cervical cancer, prognosis, immune infiltration 


\section{INTRODUCTION}

Cervical cancer remains the fourth most common cancer among women and accounts for 527,624 new diagnosed cases and 265,672 deaths in 2018 (Bray et al. (2018)). Cervical cancer continues to be the first or second leading cause of cancer-related death among women for many low- and middle-income countries (LMICs) (Wang et al. (2018)). Persistent HPV infection, especially types
16 and 18, is a high-risk factor but not the only one for cervical cancer (Revathidevi et al. (2020)). Host genetic factors may also be involved in tumor development. The major treatments for cervical cancer patients include surgery, chemotherapy, and radiotherapy. For patients with early-stage cervical cancer, 5-year survival is up to 91.5\%, while the treatment of advanced cervical cancer is not ideal (Luan and Wang (2018)). The median survival time of metastatic cervical cancer patients is about $8-13$ months, and the 5 -year overall

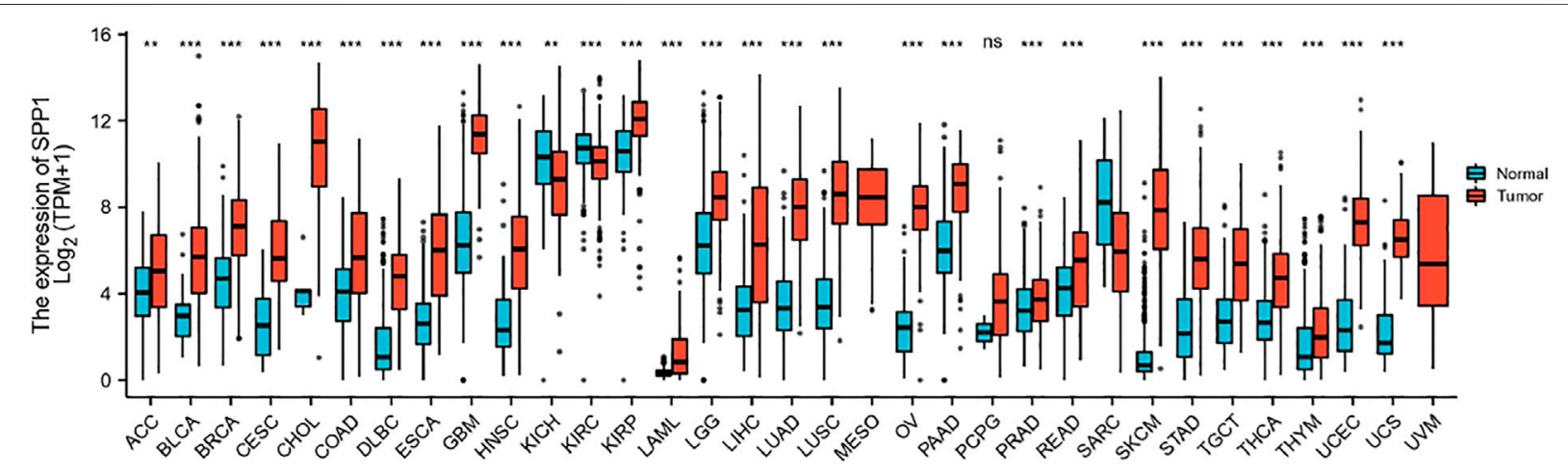

FIGURE 1 | SPP1 expression in normal and tumor tissues in TCGA and GTEx databases.

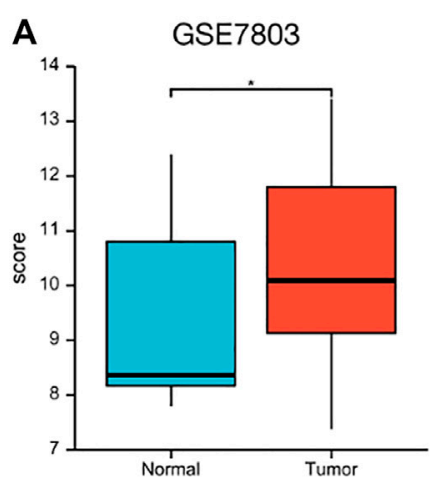

B

GSE63514

C

GSE9750
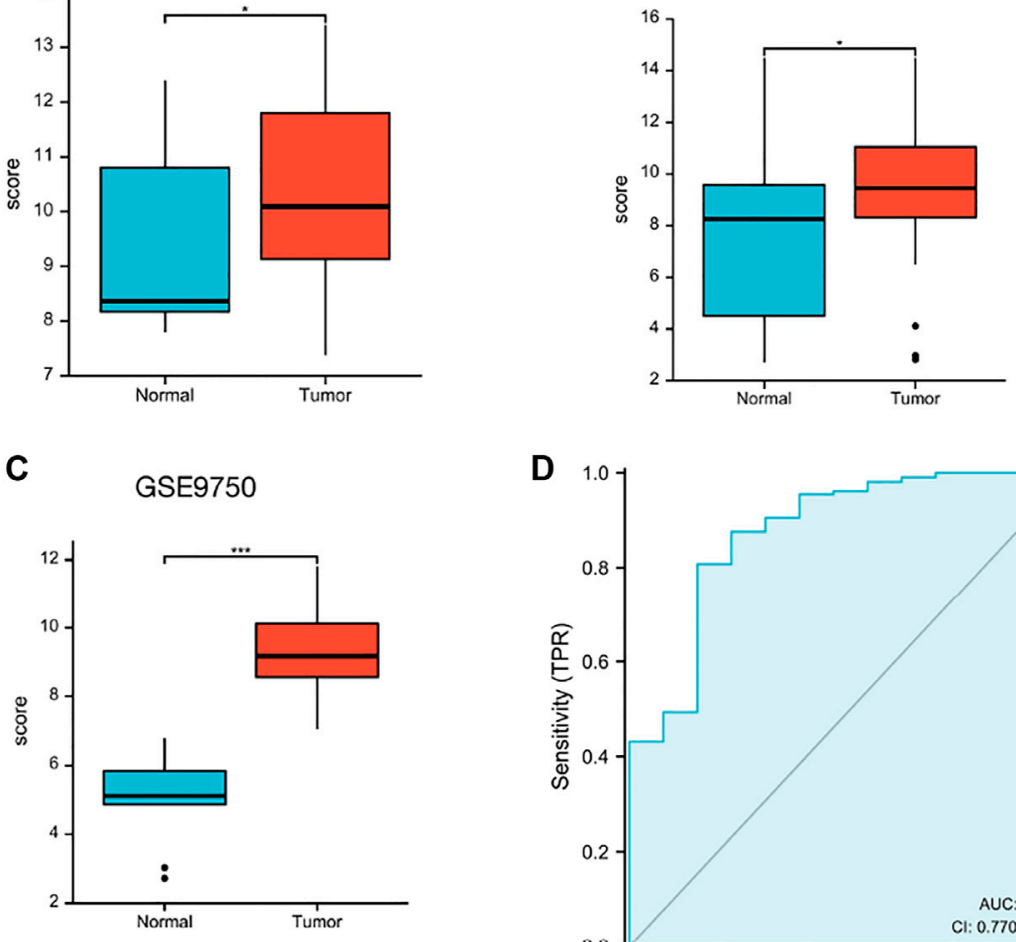

D

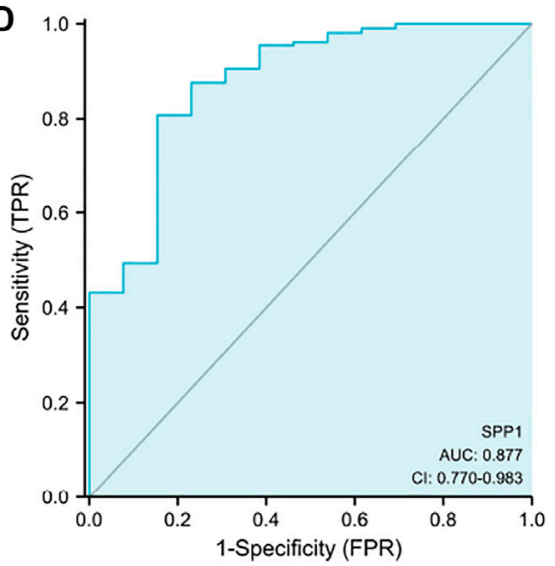

FIGURE 2 | SPP1 expression in the GEO database. (A) SPP1 expression in normal and tumor tissues in cervical cancer from GSE7803. (B) SPP1 expression in normal cervical epithelial and cervical cancer tissues from GSE63514. (C) SPP1 expression in normal cervical tissues and cervical cancer epithelial component from GSE9750. (D) ROC curve of SPP1 in cervical cancer. X-axis represents false-positive rates, and Y-axis represents true-positive rates. 
TABLE 1 | Correlation analyzed between SPP1 expression and clinicopathologic characteristics in cervical cancer based on TCGA database.

\begin{tabular}{|c|c|c|c|}
\hline Characteristic & Low expression of SPP1 & $\begin{array}{c}\text { High } \\
\text { expression of SPP1 }\end{array}$ & $p$ value \\
\hline N & 153 & 153 & \\
\hline $\mathrm{T} 1$ & $82(33.7 \%)$ & 58 (23.9\%) & \\
\hline $\mathrm{T} 2$ & $31(12.8 \%)$ & $41(16.9 \%)$ & \\
\hline T3 & $6(2.5 \%)$ & $15(6.2 \%)$ & \\
\hline T4 & $4(1.6 \%)$ & $6(2.5 \%)$ & \\
\hline N1 & 27 (13.8\%) & $34(17.4 \%)$ & \\
\hline M stage, n (\%) & & & 0.699 \\
\hline MO & $55(43.3 \%)$ & $61(48 \%)$ & \\
\hline M1 & $4(3.1 \%)$ & $7(5.5 \%)$ & \\
\hline Clinical stage, n (\%) & & & 0.020 \\
\hline Stage I & $95(31.8 \%)$ & 67 (22.4\%) & \\
\hline No & $63(20.6 \%)$ & 59 (19.3\%) & \\
\hline Yes & $90(29.4 \%)$ & $94(30.7 \%)$ & \\
\hline Primary therapy outcome, $\mathrm{n}(\%)$ & & & 0.106 \\
\hline $\mathrm{PD}$ & $7(3.2 \%)$ & $16(7.3 \%)$ & \\
\hline SD & $2(0.9 \%)$ & $4(1.8 \%)$ & \\
\hline PR & $4(1.8 \%)$ & $4(1.8 \%)$ & \\
\hline $\mathrm{CR}$ & $101(46.1 \%)$ & $81(37 \%)$ & \\
\hline Race, n (\%) & & & 0.444 \\
\hline Asian & 12 (4.6\%) & $8(3.1 \%)$ & \\
\hline Black or African American & $13(5 \%)$ & $18(6.9 \%)$ & \\
\hline White & $106(40.6 \%)$ & $104(39.8 \%)$ & \\
\hline Histologic type, n (\%) & & & $<0.001$ \\
\hline Adenosquamous & $40(13.1 \%)$ & $13(4.2 \%)$ & \\
\hline
\end{tabular}

survival rate is only around 16.5\% (Ferlay et al. (2013); van Meir et al. (2014)). Therefore, it is urgent to find more accurate biomarkers for early detection of cervical cancer and monitoring the disease progression.

Secreted phosphoprotein $1(S P P 1)$ is a secreted multifunctional phosphoprotein located in 4q13 with seven exons and six introns. $S P P 1$, also known as osteopontin-like protein or early T-lymphocyte activation 1 protein, is a member of the small integrin-binding ligand $\mathrm{N}$-linked glycoprotein (SIBLING) family which can specifically bind and activate matrix metalloproteinases (MMPs) in cancer (Su et al. (2020)). Its main biological functions are involved in immune response, biomineralization, and tissue remodeling. SPP1 is also related to the growth, proliferation, migration, apoptosis, and chemotaxis of cells. Previous studies have proven that SPP1 is overexpressed in a variety of cancers and can be used to predict the adverse consequences, including ovarian cancer (Zeng et al. (2018)), glioblastoma (Kijewska et al. (2017)), hepatocellular carcinoma (Wang et al. (2019)), and gastric cancer (Song et al.
(2019)). Recently, the relationship between the expression of SPP1 and chemotherapy resistance, such as prostate cancer and hepatocellular carcinoma, has also attracted the attention of researchers (Liu et al. (2016); Pang et al. (2019)), while no study has explored the correlation between SPP1 and cervical cancer. Therefore, our study aimed to explore the expression of SPP1 in cervical cancer tissues and its potential clinical values.

In our research, we utilized the cervical cancer RNA-seq data from The Cancer Genome Atlas (TCGA), Gene Expression Omnibus (GEO), and Genotype-Tissue Expression databases to compare the differential expression of SPP1 between normal cervical tissues and cervical cancer samples. Next, we investigated the relationship between SPP1 expression levels and clinical pathological features of cervical cancer. Furthermore, we explored the prognostic value of SPP1 in cervical cancer. Besides, we performed gene enrichment analysis to reveal its potential functions. Finally, we analyzed the relationship between SPP1 expression and immune infiltration and comprehensively 
TABLE 2 | SPP1 expression associated with clinicopathologic characteristics by logistic regression.

Characteristic

T stage (T2 and T3 and T4 vs. T1)

$\mathrm{N}$ stage (N1 vs. NO)

M stage (M1 vs. MO)

Clinical stage (Stage II and Stage III and Stage IV vs. Stage I)

Primary therapy outcome (SD and PR and CR vs. PD)

Histologic type (squamous cell carcinoma vs. adenosquamous)

Age (>50 vs. $\leq 50$ years)

Radiation therapy (yes vs. no)

Histologic grade (G2 and G3 and G4 vs. G1)
Total (N)

243

195

127

299

219

306

306

306

274
Odds ratio (OR)

2.138 (1.278-3.609)

$1.507(0.821-2.786)$

$1.578(0.451-6.294)$

2.051 (1.295-3.269)

$0.364(0.135-0.893)$

$3.812(1.993-7.732)$

$1.743(1.097-2.787)$

$1.115(0.706-1.765)$

$1.052(0.411-2.731)$ p value

0.004

0.187

0.485

0.002

0.033

$<0.001$

0.019

0.641

0.916

TABLE 3 | Univariate and multivariate Cox analyses of prognostic factors in cervical cancer.

\begin{tabular}{|c|c|c|c|c|c|}
\hline \multirow[t]{2}{*}{ Characteristic } & \multirow[t]{2}{*}{ Total (N) } & \multicolumn{2}{|c|}{ Univariate analysis } & \multicolumn{2}{|c|}{ Multivariate analysis } \\
\hline & & $\begin{array}{c}\text { Hazard } \\
\text { ratio }(95 \% \mathrm{Cl})\end{array}$ & $p$ value & $\begin{array}{c}\text { Hazard } \\
\text { ratio }(95 \% \mathrm{Cl})\end{array}$ & $p$ value \\
\hline T stage (T2 and T3 and T4 vs. T1) & 243 & $1.906(1.085-3.348)$ & 0.025 & $1.193(0.419-3.395)$ & 0.741 \\
\hline N stage (N1 vs. N0) & 195 & $2.844(1.446-5.593)$ & 0.002 & $3.117(1.517-6.403)$ & 0.002 \\
\hline M stage (M1 vs. M0) & 127 & $3.555(1.187-10.641)$ & 0.023 & & \\
\hline TP53 (high vs. low) & 306 & $0.854(0.537-1.356)$ & 0.503 & & \\
\hline Clinical stage (Stage II and Stage III and Stage IV vs. Stage I) & 299 & $1.462(0.920-2.324)$ & 0.108 & $0.464(0.160-1.345)$ & 0.157 \\
\hline Radiation therapy (yes vs. no) & 306 & $1.172(0.694-1.981)$ & 0.553 & & \\
\hline Race (Black or African American and White vs. Asian) & 261 & $1.537(0.374-6.317)$ & 0.552 & & \\
\hline Age (>50 vs. $\leq 50$ years) & 306 & 1.289 (0.810-2.050) & 0.284 & $0.658(0.298-1.452)$ & 0.299 \\
\hline Histologic type (squamous cell carcinoma vs. adenosquamous) & 306 & $1.033(0.543-1.969)$ & 0.920 & & \\
\hline Histologic grade (G2 and G3 vs. G1) & 273 & $1.212(0.378-3.882)$ & 0.746 & & \\
\hline SPP1 (high vs. low) & 306 & $1.686(1.046-2.719)$ & 0.032 & $2.207(1.019-4.777)$ & 0.045 \\
\hline
\end{tabular}

The value in bold indicates that $p$ is less than 0.05 , which is meaningful.

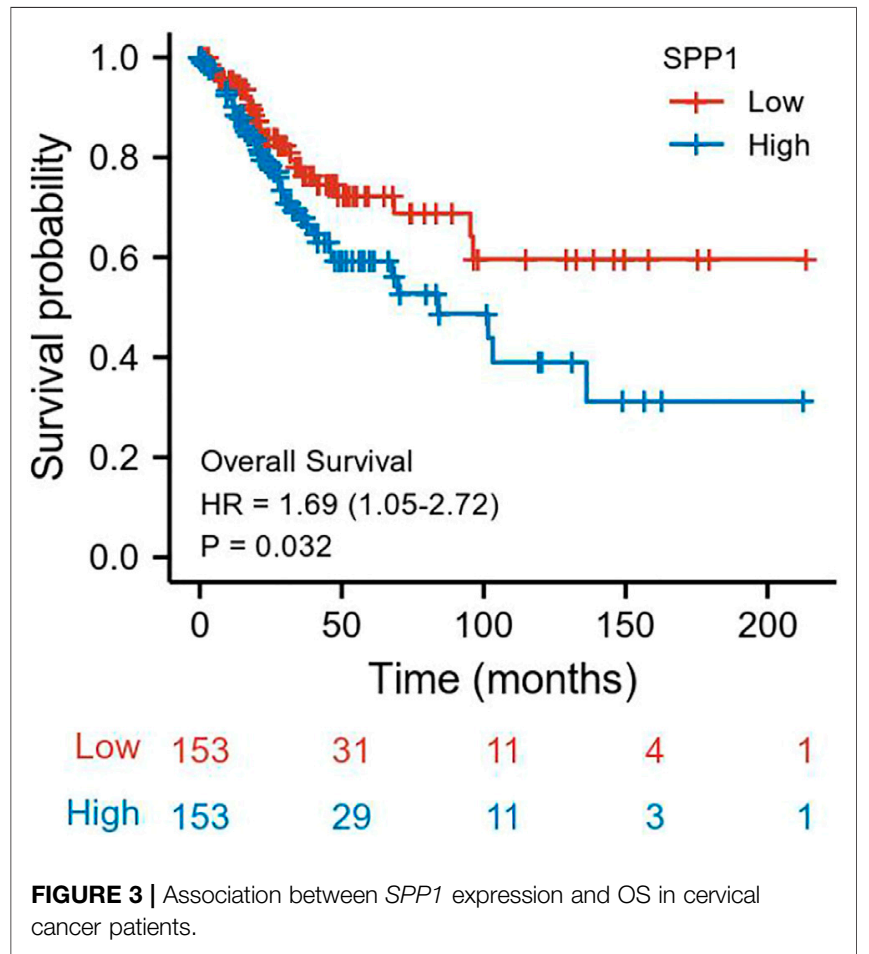

explored its mechanism in inducing and promoting cervical cancer.

\section{MATERIALS AND METHODS}

\subsection{RNA Sequencing Data Collection and Analysis}

To evaluate the SPP1 expression level in pan-cancer, we downloaded data from the UCSC Xena (https:// xenabrowser.net/datapages/). We selected samples from the TCGA database for the analysis of SPP1 expression in tumor tissues, while the combined analysis of TCGA and GenotypeTissue Expression (GTEx) databases was used for the normal tissue samples. GSE7803 (Platform: GPL96), GSE63514 (Platform: GPL570), and GSE9750 (Platform: GPL96) downloaded from GEO were used to obtain cervical cancer microarray data.

\subsection{Correlation and Gene Set Enrichment Analysis}

We used data collected from TCGA to perform correlation analysis between $S P P 1$ and other mRNAs in cervical cancer. To demonstrate the biological function of $S P P 1$, we selected 


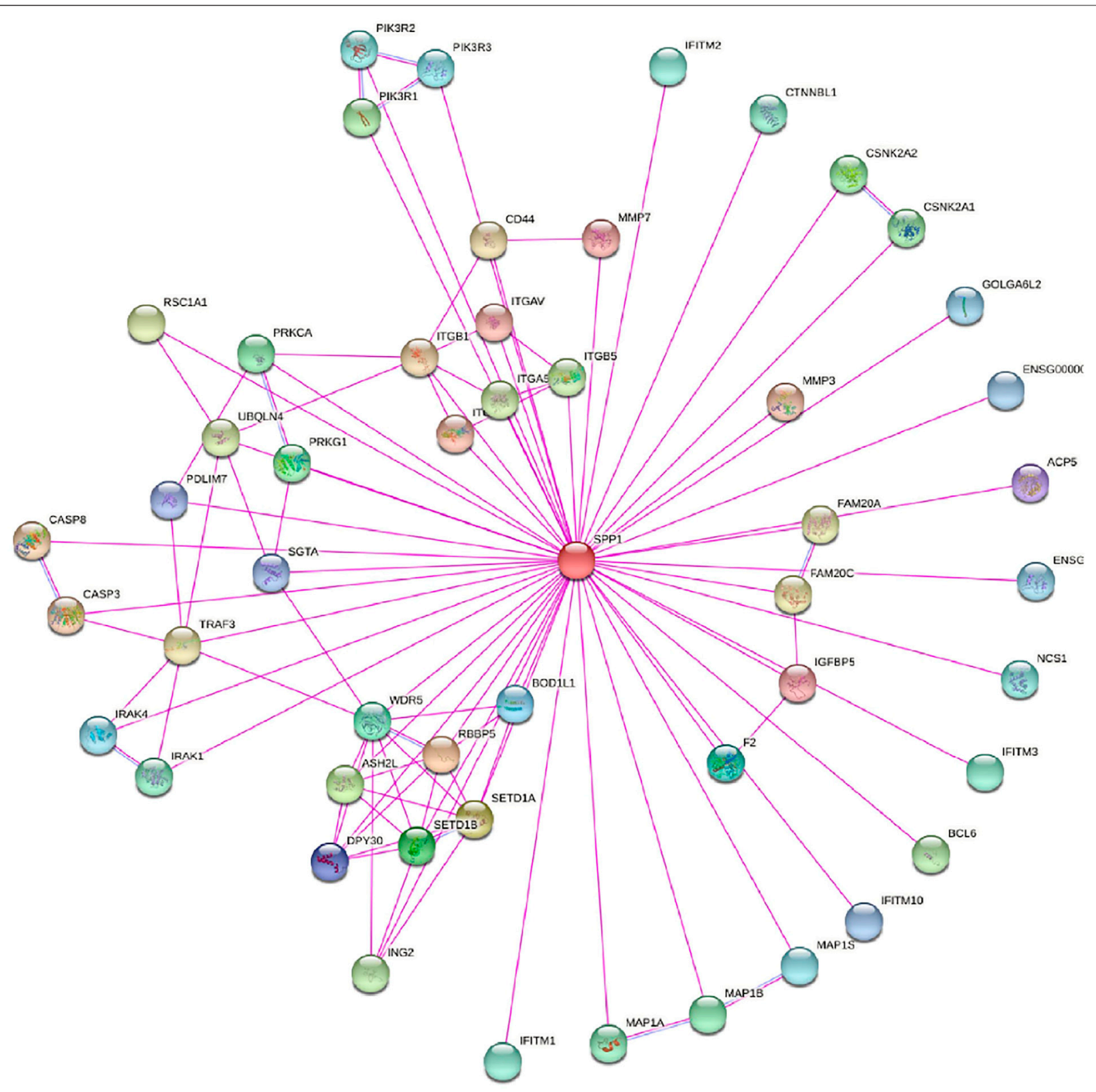

FIGURE 4 | SPP1-binding proteins obtained by the STRING tool.

the top 100 genes most positively correlated with SPP1 for enrichment analysis. EnrichGO function in the $\mathrm{R}$ package "clusterProfiler" was used to perform gene ontology (GO) enrichment, including BP, CC, and MF. Kyoto Encyclopedia of Genes and Genomes (KEGG) analysis was performed using the EnrichKEGG function of the R package "clusterProfiler."

\subsection{Survival Prognosis Analysis}

We used the R package "survival" (version 3.6) to obtain the overall survival (OS) survival plots of SPP1. Selecting the cutoff value of $50 \%$ as the dividing threshold, the cohorts were divided into high-expression and low-expression groups. To evaluate the value of SPP1 in predicting the prognosis of cervical cancer patients, we used the R package (version 3.6.3) "ROC" for analysis and "ggplot2" for visual.

\subsection{Immune Cell Infiltration Analysis}

We used the single-sample GSEA (ssGSEA) method from the R package GSVA (version 3.6) and Tumor Immune Estimation Resource (TIMER) database (http://timer.cistrome.org/) to comprehensively investigate molecular characterization of tumor-immune interactions in cervical cancer. In the literature, we examined the impact of SPP1 expression on immune cell infiltration using gene expression profiling data. To investigate the correlation between SPP1 expression and the abundances of tumor-infiltrating immune cells, $p$-values were 


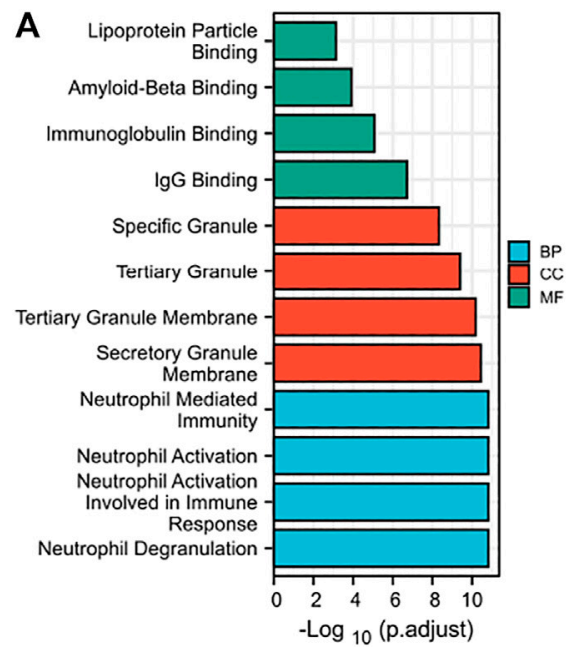

\section{B}

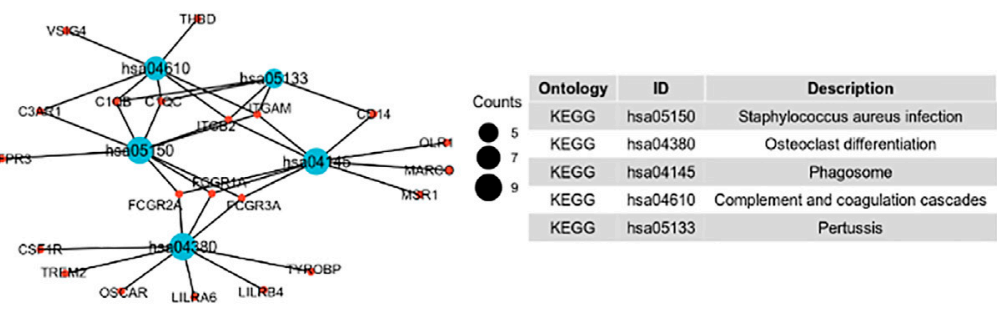

FIGURE 5 | Function and pathway enrichment analysis of SPP1 in cervical cancer. (A) Significant Gene Ontology terms (including BP, MF, and CC) of the top 100 genes most positively associated with SPP1. (B) Significant KEGG pathway of the top 100 genes most positively associated with SPP1.

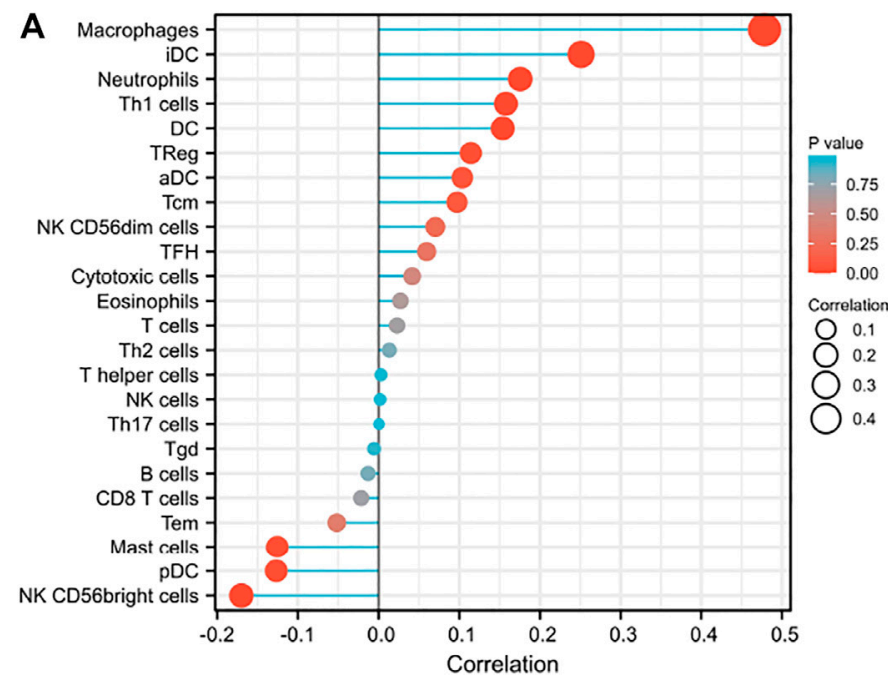

B

\begin{tabular}{lll}
\hline Molecular & cell & P value(Spearman) \\
\hline SPP1 & iDC & $<0.001$ \\
SPP1 & Macrophages & $<0.001$ \\
SPP1 & Neutrophils & 0.002 \\
& NK CD56bright & \\
SPP1 & cells & 0.003 \\
SPP1 & Th1 cells & 0.006 \\
SPP1 & DC & 0.007 \\
SPP1 & pDC & 0.027 \\
SPP1 & Mast cells & 0.028 \\
SPP1 & TReg & 0.046
\end{tabular}

FIGURE 6 | (A) Lollipop chart of SPP1 expression level in 24 immune cells. (B) The immune cell infiltration associated with SPP1 expression, P < 0.05, represents a significant result.

calculated using the Wilcoxon rank-sum and Spearman's rank correlation tests.

\section{RESULTS}

\subsection{The mRNA Expression Analysis of SPP1 in Pan-Cancer}

Data downloaded from TCGA and GTEx were used to analyze $S P P 1$ expression in 33 types of cancer. The result revealed that SPP1 was overexpressed in most cancers, including ACC, BLCA, BRCA, CESC, CHOL, COAD, DLBC, ESCA, GBM,
HNSC, KIRP, LAML, LGG, LIHC, LUAD, LUSC, OV, PAAD, PRAD, READ, SKCM, STAD, TGCT, THCA, THYM, UCEC, and UCS. However, the expression of SPP1 was low in $\mathrm{KICH}$ and KIRC (Figure 1). Furthermore, we assessed SPP1 expression in cervical cancer in the GEO database, including GSE7803 (Platform: GPL96), GSE63514 (Platform: GPL570), and GSE9750, and the results confirmed that SPP1 was overexpressed in cervical cancer tissues (Figures 2A-C). Additionally, we performed the receiver operating characteristic (ROC) curve to evaluate the feasibility of the SPP1 expression level to distinguish cervical cancer tissues from normal cervical tissues. The 


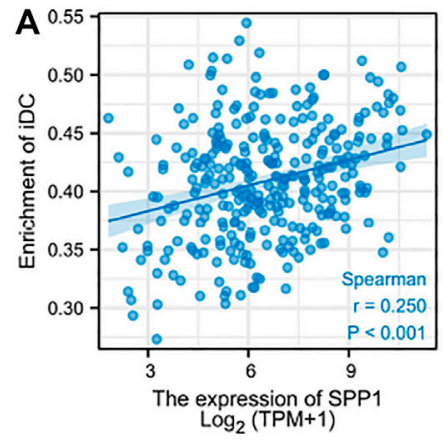

D

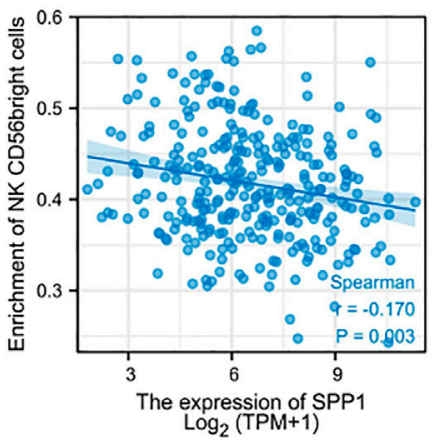

G

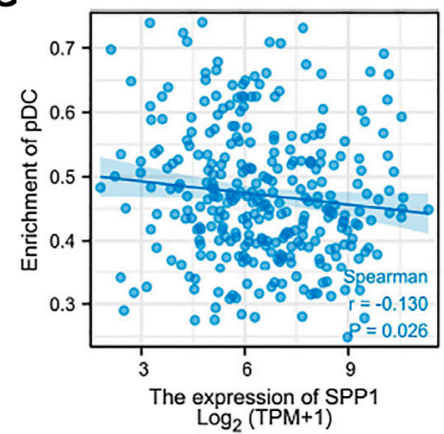

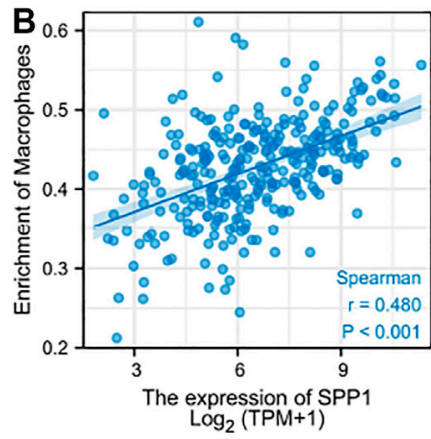

E

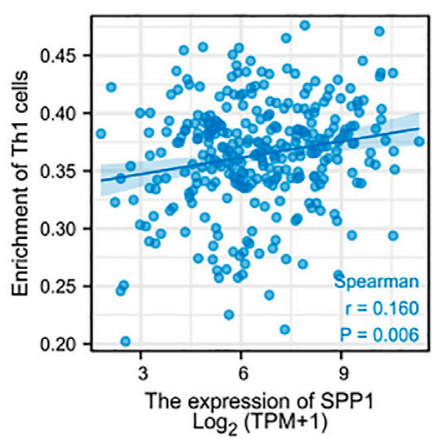

H

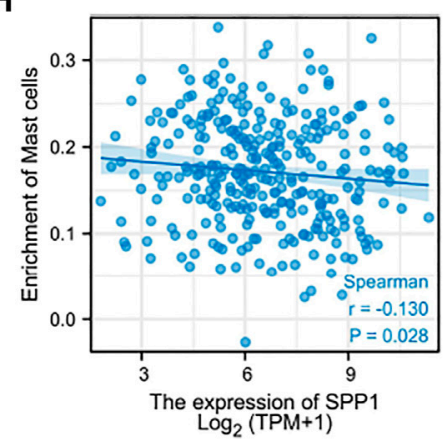

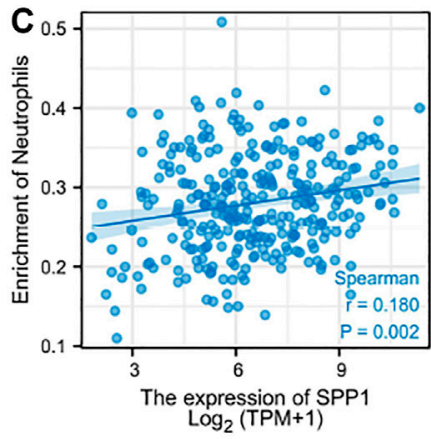

$\mathbf{F}$
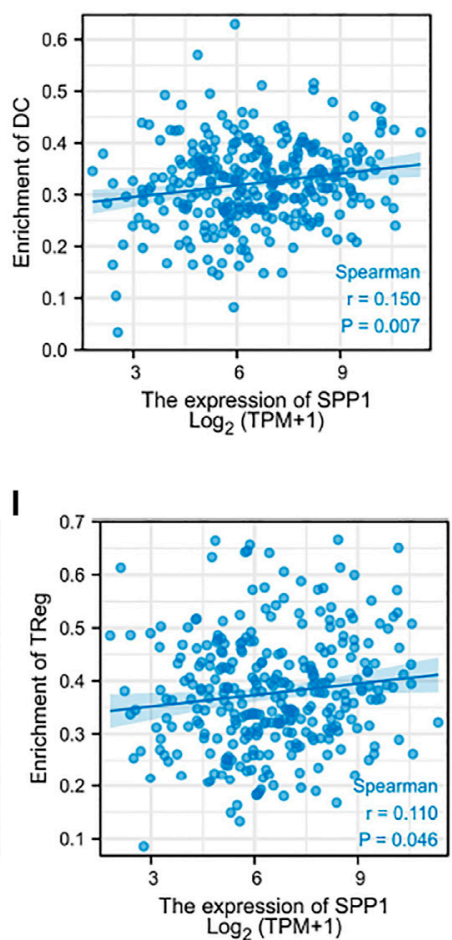

FIGURE 7 | Correlation between SPP1 expression and immune cell infiltration. (A-I) Correlation between SPP1 expression and iDC, macrophages, neutrophils, NK CD56 bright cells, Th1 cells, DC, pDC, mast cells, and Treg cells.

area under the ROC curve (AUC) was 0.877, representing the quality of the test.

\subsection{Clinical Relevance of the SPP1 Expression in Cervical Cancer Patients}

The characteristics of 306 primary cervical cancer patients with both clinical and gene expression data were downloaded from TCGA database. With the cutoff value of $50 \%$ as the dividing threshold, the patients were divided into a high-SPP1 expression group $(n=153)$ and a low-SPP1 expression group $(n=153)$. The correlation of the SPP1 expression level and patients' clinicopathologic characteristics was explored. We found that $S P P 1$ expression was significantly associated with T stage $(P=$ $0.02)$, clinical stage $(P=0.02)$, and histologic type $(P<0.001)$ by using the chi-square test or Fisher's exact test. The Wilcoxon rank-sum test revealed that $S P P 1$ expression was associated with age $(P=0.038)$ (Table 1$)$.

We conducted the logistic regression method to further analyze the relationship between the SPP 1 expression level and the clinicopathologic characteristics of cervical cancer. The results showed that the expression level of SPP1 was significantly associated with $\mathrm{T}$ stage $(P=0.004)$, clinical stage $(P=0.002)$, primary therapy outcome $(P=$ $0.033)$, histologic type $(P<0.001)$, and age $(P=0.019)$ (Table 2).

\section{Association Between SPP1 Expression and Cancer Patient Survival Prognosis}

We performed univariate and multivariate Cox analyses of overall survival (OS) in cervical cancer patients, and results are shown in Table 3. In univariate Cox analysis of SPP1, T stage 

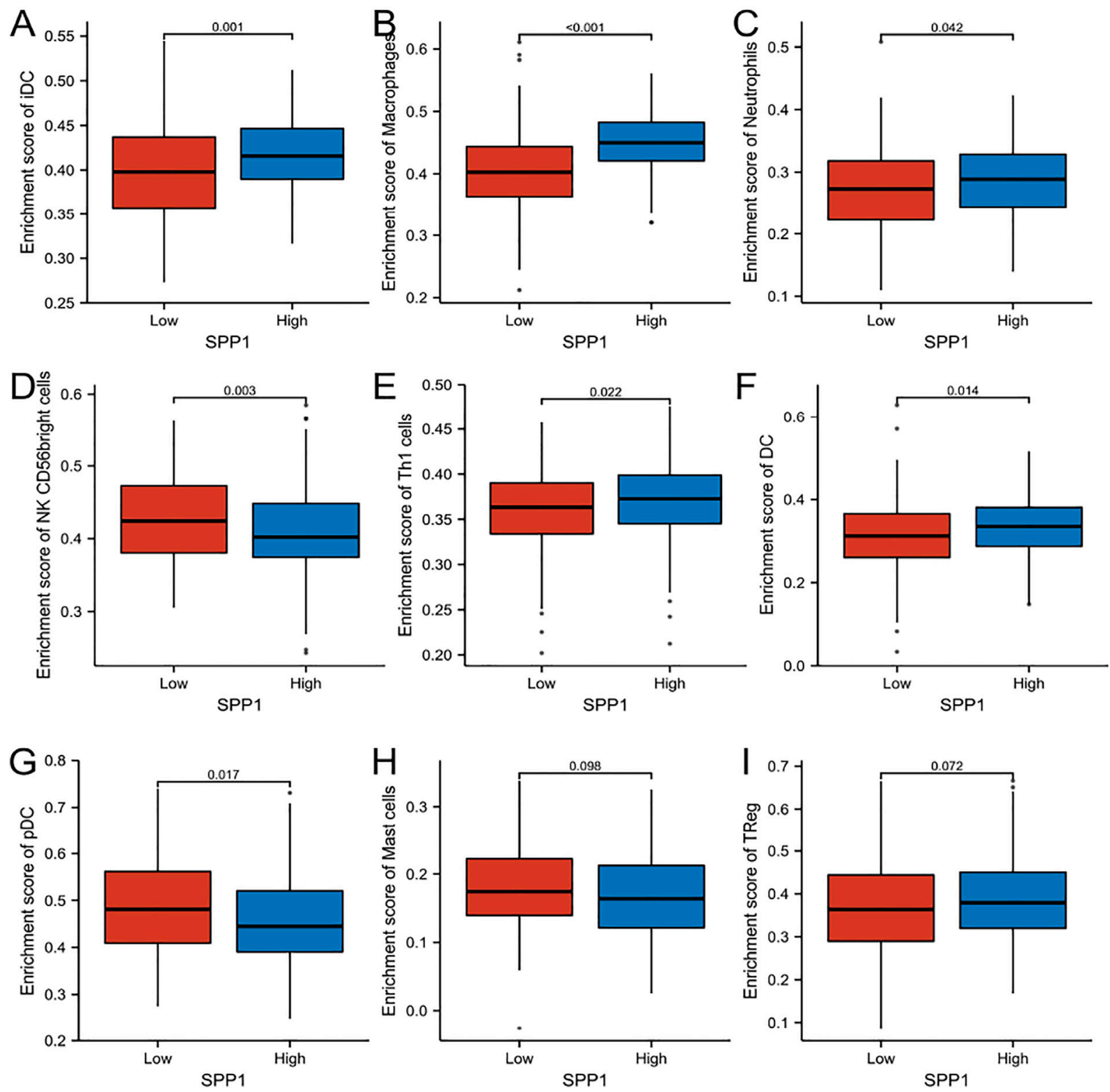

FIGURE 8 | Comparison of immune cells between high- and low-SPP1 expression groups. (A-I) Histogram showing the difference of iDC, macrophages, neutrophils, NK CD56 bright cells, Th1 cells, DC, pDC, mast cells, and Treg cell infiltration level between high-and low-SPP1 expression groups.

$(P=0.025), \mathrm{N}$ stage $(P=0.002), \mathrm{M}$ stage $(P=0.023)$, and $S P P 1$ expression $(P=0.032)$ were associated with overall survival (OS) in cervical cancer patients. In the multivariate Cox model, we found that $\mathrm{N}$ stage $(P=0.002)$ and $S P P 1$ expression $(P=$ $0.045)$ were still relevant to worse prognosis. Furthermore, we investigated the relationship between SPP1 expression and overall survival (OS) of cervical cancer patients. According to the KM plot, patients with higher SPP1 mRNA expression showed poorer prognosis than the lower group $(\mathrm{HR}=1.69$, 95\% CI: $1.05-2.72, P=0.032$ ) (Figure 3). Thus, SPP1 may become a promising prognostic biomarker for cervical cancer patients.

\subsection{Correlation and SPP1-Related Gene Enrichment Analysis}

In this study, we only considered physically binding protein interactions and obtained 50 experimental supported SPP1binding proteins from the STRING network (Figure 4). We downloaded data from TCGA database to further investigate 

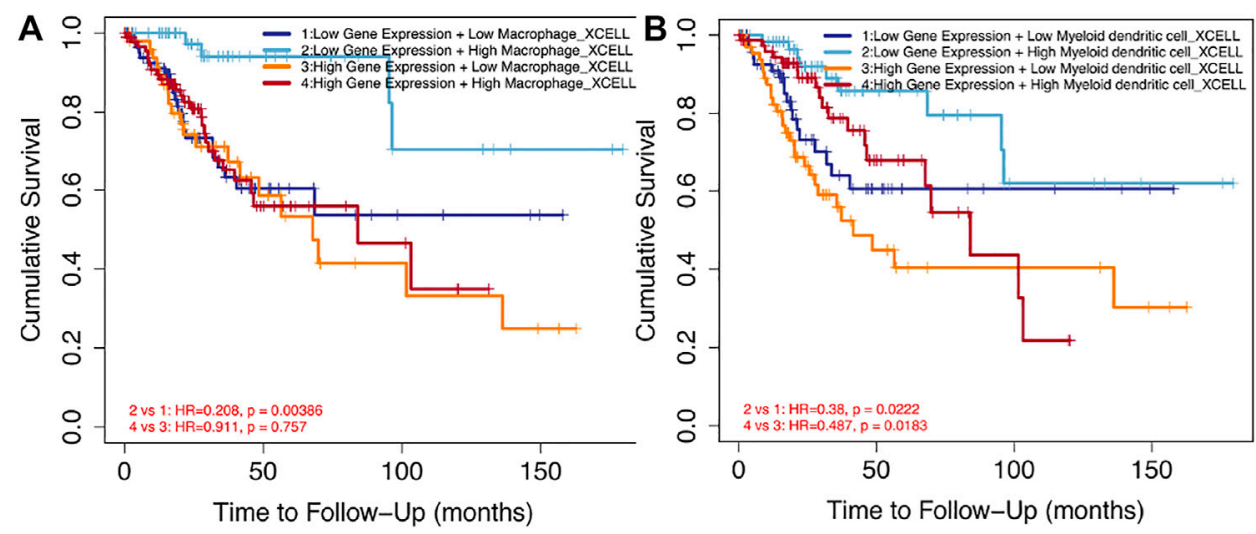

FIGURE 9 | Impact of immune cell infiltration on prognosis in cervical cancer patients. (A) Clinical survival outcome of cervical cancer patients in the highmacrophage group. (B) Clinical survival outcome of cervical cancer patients in the high-DC cell group.

the function of SPP1 and search SPP1 expression-correlated genes for related pathway analysis. We obtained the top 100 most positively correlated genes with SPP1 for GO and KEGG enrichment analysis by the "clusterProfile" $\mathrm{R}$ package. The GO analysis data showed that most of the genes were associated with neutrophil degranulation, neutrophil activation involved in immune response, neutrophil activation, and neutrophilmediated immunity (Figure 5A). The KEGG data suggested that the "phagosome" may be related to the carcinogenic mechanism of SPP1 (Figure 5B).

\subsection{Relationship Between SPP1 Expression and Immune Cell Infiltration}

Through the previous enrichment analysis, we found that SPP1 was mainly related to neutrophils and phagosomes. We hypothesized that there might be some relationship between SPP1 and immune cells. Thus, we further assessed whether the SPP1 expression level was associated with immune cell infiltration. We used ssGSEA from the $\mathrm{R}$ package with Spearman's $r$ to investigate the potential association between the SPP1 expression level and 24 types of immune cells. The result revealed that $S P P 1$ expression had significant correlation with iDC, macrophages, neutrophils, NK CD56 bright cells, Th1 cells, DC, pDC, mast cells, and Treg cells (Figure 6). Further research showed that $S P P 1$ expression was positively correlated with infiltration levels of iDC (Figure 7A) $(r=0.250, P<0.001)$, macrophages (Figure 7B) $(r=0.480, P<0.001)$, neutrophils (Figure 7C) $(r=0.180, P=$ 0.002), Th1 cells (Figure 7E) $(r=0.160, P=0.006)$, DC (Figure 7F) $(r=0.150, P=0.007)$, and Treg cells (Figure $7 \mathrm{I})(r=0.110, P=$ 0.046). In contrast, SPP1 expression was negatively correlated with that of NK CD56 bright cells (Figure 7D) $(r=-0.170, P=0.003)$, pDC (Figure 7G) $(r=-0.130, P=0.026)$ and mast cells (Figure 7H) $(r=-0.130, P=0.028)$. This prompted us to examine the relationship between the SPP1 expression level and immune infiltration. Surprisingly, we found significant differences in infiltrating immune cell levels, including iDC, macrophages, neutrophils, NK CD56 bright cells, Th1 cells, DC, and pDC $(P<0.05)$, when SPP1 expression was categorized into high and low groups (Figures
8A-G), while no significant difference in mast cells and Treg cells was noted (Figures $\mathbf{8 H}, \mathbf{I}$ ). Finally, we assessed the impact of immune cell infiltration on clinical survival outcome of cervical cancer patients by TIMER (http://timer.cistrome.org/). We found that high levels of macrophages and DC cells were associated with poor prognosis of cervical cancer patients $(P<0.05)$ (Figures 9A,B).

\section{DISCUSSION}

Invasive cervical cancer remains the leading cause of cancer death among women worldwide (Shen et al. (2020)). Thus, it is necessary to find more accurate biomarkers to detect at an early stage and monitor disease progression. According to the previous studies, SPP1 is overexpressed in various cancer types (Xu et al. (2017); Choe et al. (2018); Zhang et al. (2020)) and identified as a prognostic factor (Li et al. (2018); Chen J et al. (2019); Guo et al. (2020)), while to our knowledge, no study has explored the relationship of SPP1 expression and cervical cancer. In our study, we attempted to explore the potential mechanism of SPP1 in promoting cervical cancer and its feasibility as a molecular biomarker.

In pan-cancer analysis, we found that $S P P 1$ was upregulated in most cancer types. Further exploration revealed that higher SPP1 expression was associated with reduced overall survival (OS) in cervical cancer patients. We performed logistic regression to evaluate the relationship between the SPP1 expression level and the clinicopathologic characteristics of cervical cancer. The result showed that SPP1 was significantly correlated with clinical stages. In addition, univariate and multivariate Cox analyses indicated that SPP1 was an independent factor to predict prognosis of patients. All these aforementioned results and ROC analysis suggest that SPP1 may be a promising prognostic biomarker for cervical cancer patients.

The tumor microenvironment (TME), composed of various types of immune cells, played an important role in tumor progression, metastasis, and treatment resistance (Usui et al. (2016)). The composition of tumor-infiltrating immune cells strongly influenced the tumor microenvironment and the 
behavior of the tumor. Our gene enrichment analysis revealed that the main biological function of SPP1 was mainly involved in immune response. We next confirmed that SPP1 expression correlated with immune cell infiltration. Hence, we hypothesized that SPP1 may affect the tumor microenvironment by changing proportions of specific immune cell types, thereby promoting tumor progression and metastasis. It was, indeed, the case that $S P P 1$ had recently been shown to be an important component in maintaining the tumor microenvironment in AML (Ruvolo et al. (2019)). Our research demonstrated the significant positive correlation between macrophages and the expression of SPP1. Macrophages are important components of the tumor microenvironment, and tumor-associated macrophages play complex roles in cancer pathophysiology (Gibson et al. (2019)). A previous study found that SPP1 was involved in the function, migration, and differentiation of macrophages (Zhang et al. (2017); Wei et al. (2019); Jaitin et al. (2019); Srirussamee et al. (2019)). A recent study also showed that SPP1 was essential for M2-like macrophage, the tumor-associated macrophage, and promoted tumor growth (Chen P et al. (2019)). Furthermore, we found that the increased level of macrophages and DC infiltration were correlated with poor prognosis. Our results were supported by the findings of similar studies about this topic (Long et al. (2016); Ndiaye et al. (2019)). Certainly, the tumor microenvironment had a high level of complexity in its regulation; other immune cell types in the tumor microenvironment may also influence tumor cell survival, including iDC, neutrophils, NK CD56 bright cells, Th1 cells, DC, and pDC. Future studies were needed to further explore the relationship between SPP1 expression and these cells.

In conclusion, we demonstrated that SPP1 expression was upregulated in cervical cancer and significantly related to poor survival outcome. In addition to this, SPP1 might participate in the occurrence and development of cervical cancer by influencing the infiltration level of immune cells. Therefore, our study revealed the role of SPP1 in cervical cancer and identified a promising prognostic biomarker.

Although our study is the first work to explore the relationship between SPP1 expression and cervical cancer, it also has some limitations. First, all of the data analyzed by bioinformatics methods in this study were downloaded directly from public databases, so it requires further validation by experimental investigations; second, the number of normal samples used as controls was considerably different from that of patients with tumor in the TCGA database; therefore, further studies based on

\section{REFERENCES}

Bray, F., Ferlay, J., Soerjomataram, I., Siegel, R. L., Torre, L. A., and Jemal, A. (2018). Global Cancer Statistics 2018: Globocan Estimates of Incidence and Mortality Worldwide for 36 Cancers in 185 Countries. CA Cancer J. Clin. 68, 394-424. doi:10.3322/caac.21492

Chen J, J., Hou, C., Zheng, Z., Lin, H., Lv, G., and Zhou, D. (2019). Identification of Secreted Phosphoprotein 1 (Spp1) as a Prognostic Factor in Lower-Grade Gliomas. World Neurosurg. 130, e775. doi:10.1016/j.wneu.2019.06.219

Chen P, P., Zhao, D., Li, J., Liang, X., Li, J., Chang, A., et al. (2019). Symbiotic Macrophage-Glioma Cell Interactions Reveal Synthetic an equal balance of sample size are necessary. Third, further validation studies with a long-term follow-up and larger cohorts of patients are needed to definitely validate SPP1 as an OS predictor. Last but not least, our study laid the foundation for detailed studies of the correlation between SPP1 and the tumorassociated immune microenvironment. However, more studies are required to explore the hypothesis in depth.

\section{STATEMENT}

The cervical cancer cell lines (Siha and Hela) present in this study were obtained from the Scientific Research Center of Zhongnan Hospital of Wuhan University. And normal cervical epithelial cell (END1) was donated by Wuhan University Basic Medical College.

\section{DATA AVAILABILITY STATEMENT}

Publicly available datasets were analyzed in this study. These data can be found freely from TCGA data portal (https://portal. gdc.cancer.gov/) and GEO database (https://www.ncbi.nlm.nih. gov/geo/).

\section{AUTHOR CONTRIBUTIONS}

$\mathrm{KZ}$ and WZ contributed to the study conception and design. Material preparation, data collection, and analysis were performed by $\mathrm{KZ}$ and $\mathrm{ZM}$. KZ contributed to the literature search. The first draft of the manuscript was written by $K Z$, and all authors commented on previous versions of the manuscript. WZ reviewed the article and gave suggestions on the revision of the article. All authors read and approved the final manuscript.

\section{FUNDING}

Our research was supported by the project of improving the ability of diagnosis and treatment of difficult diseases in Zhongnan Hospital of Wuhan University. The project number is ZLYNXM202019.

Lethality in Pten-Null Glioma. Cancer cell 35, 868-884. doi:10.1016/ j.ccell.2019.05.003

Choe, E. K., Yi, J. W., Chai, Y. J., and Park, K. J. (2018). Upregulation of the Adipokine Genes Adipor1 and Spp1 Is Related to Poor Survival Outcomes in Colorectal Cancer. J. Surg. Oncol. 117, 1833-1840. doi:10.1002/jso.25078

Ferlay, J., Steliarova-Foucher, E., Lortet-Tieulent, J., Rosso, S., Coebergh, J. W. W. Comber, H., et al. (2013). Cancer Incidence and Mortality Patterns in Europe: Estimates for 40 Countries in 2012. Eur. J. Cancer 49 (6), 1374-1403. doi:10.1016/j.ejca.2012.12.027

Gibson, E. M., Nagaraja, S., Ocampo, A., Tam, L. T., Wood, L. S., Pallegar, P. N., et al. (2019). Methotrexate Chemotherapy Induces Persistent Tri-glial Dysregulation that Underlies Chemotherapy-Related Cognitive Impairment. Cell 176, 43-55. doi:10.1016/j.cell.2018.10.049 
Guo, Z., Huang, J., Wang, Y., Liu, X. P., Li, W., Yao, J., et al. (2020). Analysis of Expression and its Clinical Significance of the Secreted Phosphoprotein 1 in Lung Adenocarcinoma. Front. Genet. 11, 547. doi:10.3389/fgene.2020.00547

Jaitin, D. A., Adlung, L., Thaiss, C. A., Weiner, A., Li, B., Descamps, H., et al. (2019). Lipid-associated Macrophages Control Metabolic Homeostasis in a Trem2dependent Manner. Cell 178, 686-698. doi:10.1016/j.cell.2019.05.054

Kijewska, M., Kocyk, M., Kloss, M., Stepniak, K., Korwek, Z., Polakowska, R., et al. (2017). The Embryonic Type of Spp1 Transcriptional Regulation Is Re-activated in Glioblastoma. Oncotarget 8, 16340-16355. doi:10.18632/oncotarget.14092

Li, S., Yang, R., Sun, X., Miao, S., Lu, T., Wang, Y., et al. (2018). Identification of Spp1 as a Promising Biomarker to Predict Clinical Outcome of Lung Adenocarcinoma Individuals. Gene 679, 398-404. doi:10.1016/j.gene.2018.09.030

Liu, G., Fan, X., Tang, M., Chen, R., Wang, H., Jia, R., et al. (2016). Osteopontin Induces Autophagy to Promote Chemo-Resistance in Human Hepatocellular Carcinoma Cells. Cancer Lett. 383 (2), 171-182. doi:10.1016/ j.canlet.2016.09.033

Long, K. B., Gladney, W. L., Tooker, G. M., Graham, K., Fraietta, J. A., and Beatty, G. L. (2016). IFN $\gamma$ and CCL2 Cooperate to Redirect Tumor-Infiltrating Monocytes to Degrade Fibrosis and Enhance Chemotherapy Efficacy in Pancreatic Carcinoma. Cancer Discov. 6 (4), 400-413. doi:10.1158/21598290.cd-15-1032

Luan, X., and Wang, Y. (2018). Lncrna Xloc_006390 Facilitates Cervical Cancer Tumorigenesis and Metastasis as a Cerna against Mir-331-3p and Mir-338-3p. J. Gynecol. Oncol. 29, e95. doi:10.3802/jgo.2018.29.e95

Ndiaye, P. D., Dufies, M., Giuliano, S., Douguet, L., Grépin, R., Durivault, J., et al. (2019). Vegfc Acts as a Double-Edged Sword in Renal Cell Carcinoma Aggressiveness. Theranostics 9, 661-675. doi:10.7150/thno.27794

Pang, X., Xie, R., Zhang, Z., Liu, Q., Wu, S., and Cui, Y. (2019). Identification of Sppl as an Extracellular Matrix Signature for Metastatic Castration-Resistant Prostate Cancer. Front. Oncol. 9, 924. doi:10.3389/fonc.2019.00924

Revathidevi, S., Murugan, A. K., Nakaoka, H., Inoue, I., and Munirajan, A. K. (2020). Apobec: A Molecular Driver in Cervical Cancer Pathogenesis. Cancer Lett. 496, 104-116. doi:10.1016/j.canlet.2020.10.004

Ruvolo, P. P., Hu, C. W., Qiu, Y., Ruvolo, V. R., Go, R. L., Hubner, S. E., et al. (2019). Lgals3 Is Connected to Cd74 in a Previously Unknown Protein Network that Is Associated with Poor Survival in Patients with Aml. EBioMedicine 44, 126-137. doi:10.1016/j.ebiom.2019.05.025

Shen, S., Zhang, S., Liu, P., Wang, J., and Du, H. (2020). Potential Role of Micrornas in the Treatment and Diagnosis of Cervical Cancer. Cancer Genet. 248-249, 25-30. doi:10.1016/j.cancergen.2020.09.003

Song, S. Z., Lin, S., Liu, J. N., Zhang, M. B., Du, Y. T., Zhang, D. D., et al. (2019). Retracted : Targeting of SPP1 by microRNA-340 Inhibits Gastric Cancer Cell Epithelial-Mesenchymal Transition through Inhibition of the PI3K/AKT Signaling Pathway. J. Cel Physiol. 234, 18587-18601. doi:10.1002/jcp.28497

Srirussamee, K., Mobini, S., Cassidy, N. J., and Cartmell, S. H. (2019). Direct Electrical Stimulation Enhances Osteogenesis by Inducing Bmp2 and Spp1 Expressions from Macrophages and Preosteoblasts. Biotechnol. Bioeng. 116, 3421-3432. doi:10.1002/bit.27142

Su, X., Xu, B., Zhou, D.-L., Ye, Z., He, H., Yang, X. H., et al. (2020). Polymorphisms in Matricellular Spp1 and Sparc Contribute to Susceptibility to Papillary Thyroid Cancer. Genomics 112, 4959. doi:10.1016/j.ygeno.2020.09.018
Usui, T., Sakurai, M., Enjoji, S., Kawasaki, H., Umata, K., Ohama, T., et al. (2016) Establishment of a Novel Model for Anticancer Drug Resistance in ThreeDimensional Primary Culture of Tumor Microenvironment. Stem Cell Int. 2016, 7053872. doi:10.1155/2016/7053872

van Meir, H., Kenter, G., Burggraaf, J., Kroep, J., Welters, M., Melief, C., et al. (2014). The Need for Improvement of the Treatment of Advanced and Metastatic Cervical Cancer, the Rationale for Combined ChemoImmunotherapy. Anticancer Agents Med. Chem. 14 (2), 190-203. doi:10.2174/18715206113136660372

Wang, L., Zhao, Y., Wang, Y., and Wu, X. (2018). The Role of Galectins in Cervical Cancer Biology and Progression. Biomed. Res. Int. 2018, 2175927. doi:10.1155/ 2018/2175927

Wang, J., Hao, F., Fei, X., and Chen, Y. (2019). SPP1 Functions as an Enhancer of Cell Growth in Hepatocellular Carcinoma Targeted by Mir-181c. Am. J. Transl. Res. 11 (11), 6924-6937.

Wei, J., Marisetty, A., Schrand, B., Gabrusiewicz, K., Hashimoto, Y., Ott, M., et al. (2019). Osteopontin Mediates Glioblastoma-Associated Macrophage Infiltration and Is a Potential Therapeutic Target. J. Clin. Invest. 129, 137-149. doi:10.1172/JCI121266

Xu, C., Sun, L., Jiang, C., Zhou, H., Gu, L., Liu, Y., et al. (2017). Spp1, Analyzed by Bioinformatics Methods, Promotes the Metastasis in Colorectal Cancer by Activating Emt Pathway. Biomed. Pharmacother. 91, 1167-1177. doi:10.1016/ j.biopha.2017.05.056

Zeng, B., Zhou, M., Wu, H., and Xiong, Z. (2018). Spp1 Promotes Ovarian Cancer Progression via Integrin $\beta 1 /$ fak/akt Signaling Pathway. Onco. Targets Ther. 11, 1333-1343. doi:10.2147/ott.s154215

Zhang, Y., Du, W., Chen, Z., and Xiang, C. (2017). Upregulation of Pd-L1 by Spp1 Mediates Macrophage Polarization and Facilitates Immune Escape in Lung Adenocarcinoma. Exp. Cel Res. 359, 449-457. doi:10.1016/ j.yexcr.2017.08.028

Zhang, Q., Li, L., Lai, Y., and Zhao, T. (2020). Silencing of Spp1 Suppresses Progression of Tongue Cancer by Mediating the Pi3k/akt Signaling Pathway. Technol. Cancer Res. Treat. 19, 1533033820971306. doi:10.1177/ 1533033820971306

Conflict of Interest: The authors declare that the research was conducted in the absence of any commercial or financial relationships that could be construed as a potential conflict of interest.

Publisher's Note: All claims expressed in this article are solely those of the authors and do not necessarily represent those of their affiliated organizations, or those of the publisher, the editors, and the reviewers. Any product that may be evaluated in this article, or claim that may be made by its manufacturer, is not guaranteed or endorsed by the publisher.

Copyright (C) 2022 Zhao, Ma and Zhang. This is an open-access article distributed under the terms of the Creative Commons Attribution License (CC BY). The use, distribution or reproduction in other forums is permitted, provided the original author(s) and the copyright owner(s) are credited and that the original publication in this journal is cited, in accordance with accepted academic practice. No use, distribution or reproduction is permitted which does not comply with these terms. 


\section{GLOSSARY}

aDC activated DC

ACC adrenocortical carcinoma

BLCA bladder urothelial carcinoma

BRCA breast invasive carcinoma

CESC cervical squamous cell carcinoma and endocervical adenocarcinoma

CHOL cholangiocarcinoma

COAD colon adenocarcinoma

DLBC lymphoid neoplasm diffuse large B-cell lymphoma

ESCA esophageal carcinoma

GBM glioblastoma multiforme

GEO Gene Expression Omnibus

GO Gene Ontology

HNSC head and neck squamous cell carcinoma

iDC immature DC

KICH kidney chromophobe

KIRC kidney renal clear cell carcinoma

KIRP kidney renal papillary cell carcinoma

KEGG Kyoto Encyclopedia of Genes and Genomes

LAML acute myeloid leukemia

LGG lower grade glioma

LIHC liver hepatocellular carcinoma
LUAD lung adenocarcinoma

LUSC lung squamous cell carcinoma

OS overall survival

OV ovarian serous cystadenocarcinoma

pDC plasmacytoid DC

PAAD pancreatic adenocarcinoma

PRAD prostate adenocarcinoma

READ rectum adenocarcinoma

SKCM skin cutaneous melanoma

STAD stomach adenocarcinoma

SPP1 secreted phosphoprotein 1

Tcm T central memory

Tem $\mathrm{T}$ effector memory

Tfh $\mathrm{T}$ follicular helper

Tgd T gamma delta.

TCGA The Cancer Genome Atlas

TGCT testicular germ cell tumor

THCA thyroid carcinoma

THYM thymoma

UCEC uterine corpus endometrial carcinoma

UCS uterine carcinosarcoma 